\title{
RELATIONS ENTRE LA POPULATION DE TRUITES COMMUNES (SALMO TRUTTA), LES MACROPHYTES ET LES PARAMĖTRES DU MILIEU SUR UN RUISSEAU
}

\author{
J. HAURY et J.L. BAGLINIËRE \\ Laboratoire d'Écologie hydrobiologique - INRA Rennes \\ 65 , route de Saint-Brieuc - 35042 RENNES Cedex
}

\section{RÉSUMÉ}

Les relations entre la population de truites(Sa/mo trutta), les peuplements macrophytiques et les caractéristiques abiotiques de l'habitat, ont été étudiées sur un ruisseau découpé en tronçons consécutifs. Les facteurs du milieu ont été analysés en été et en hiver; les phytocénoses ont été recensées en été; les populations de truites ont été échantillonnées en automne.

Le traitement des données a été réalisé par une Analyse Factorielle Multiple. La densité et la taille des truites selon l'âge et le sexe sont corrélées avec des paramètres mésologiques et avec l'abondance de certaines espèces végétales. Le premier facteur, commun aux trois compartiments, correspond au gradient "amont-aval" de l'écosystème. Le second est plus nettement marqué par les variables mésologiques et végétales.

Une ségrégation spatiale des classes d'âge, voire des sexes, s'effectue principalement en fonction de la granulométrie. Les individus les plus jeunes $(0+)$ et les plus vieux (2 ans et plus) semblent avoir une plus grande stabilité spatiale contrairement aux individus $1+$, ce qui s'exprime par une corrélation plus grande entre effectif et croissance.

Les résultats sur l'écologie des macrophytes aquatiques et sur les relations entre l'abondance de certaines plantes ripicoles et les paramètres mésologiques permettent d'utiliser les végétaux comme des descripteurs synthétiques de l'habitat de la truite dans les conditions d'étude de ce milieu.

Mots-clés : Truite commune, Habitat, Macrophytes, Méthodologie, Analyse Factorielle Multiple, Bretagne.

\section{RELATIONSHIPS BETWEEN THE BROWN TROUT (SALMO TRUTTA L.) POPULATION, THE MACROPHYTE SPECIES AND THE FACTORS OF THE ABIOTIC HABITAT IN A BROOK}

\section{ABSTRACT}

The relationships between a trout population (Salmo trutta), the macrophyte species and the factors of the abiotic habitat have been studied in consecutive stretches of a brook. These abiotic factors have been recorded in summer and in winter; the vegetation have been surveyed in summer ; the populations of trout have been sampled in autumn.

The treatment of data was done with an "Analyse Factorielle Multiple (Multiple Factor Analysis)". The density and the size of the trouts according to their age and sex have been correlated with abiotic parameters and with the abundance of some macrophyte species.

The first factor describing at the same time the three compartments of the ecosystem is the "upstream-downstream" gradient. The second one is mainly due to mesologic and botanical parameters.

Spatial segregation of the age classes, indeed of sexes, depends on bed substratum. The youngest trouts $(0+)$ and the oldest ( 2 years or more) seem to have a good spatial stability while $1+$ individuals haven't. So, for young and old fish, there is a close correlation between number and size.

The results of the ecology of aquatic macrophytes and the relations between abundance of some bankside plants and the mesological parameters allow to use vegetation as complementary descriptive parameter of trout habitats, in that study.

Keywords : Brown Trout, Habitat, Macrophytes, Methods, "Analyse Factorielle Multiple", Brittany. 


\section{INTRODUCTION}

Dans l'étude des populations de truites, la connaissance de l'habitat est souvent considérée comme primordiale (MILNER et al., 1985). Pour définir et décrire cet habitat, de nombreux travaux ont été réalisés (CHAMPIGNEULLE, 1978 ; BAGLINIERE et CHAMPIGNEULLE, 1982). Toutefois, les méthodes proposées par ces auteurs sont souvent élaborées sur des secteurs-tests bien différenciés et ne prennent en compte que les meilleurs descripteurs abiotiques de l'habitat des truites (vitesse de courant, granulométrie du fond et hauteur d'eau). Que se passe-t-il lorsque l'on considère un milieu dans son intégralité et si l'on analyse d'autres compartiments biotiques du cours d'eau, comme les macrophytes?

Pour répondre à ces questions, une étude a été entreprise durant une année sur le cours principal d'un petit ruisseau de Bretagne où, depuis 1980, une analyse séparée des différents compartiments de l'écosystème a été réalisée (populations de truites : BAGLINIERE et al., 1987; MAISSE et al., 1987 ; BAGLINIERE et al., 1989; mésologie et macrophytes : HAURY, 1982 et 1985).

Présentement, dans cette étude conjointe, il s'agit :

- de déterminer la localisation des différentes classes d'âge de truites en fonction des paramètres descriptifs de l'habitat,

- d'envisager les facteurs de distribution des phytocénoses et d'examiner comment les macrophytes peuvent être utilisées comme des "intégrateurs" des paramètres mésologiques analytiques qui déterminent I'habitat trutticole,

- de mettre en évidence les facteurs statistiques communs à la structure de chaque compartiment de l'écosystème.

Cette tentative d'approche globale est rendue possible par l'emploi d'une nouvelle méthode d'analyse multidimensionnelle : I'Analyse Factorielle Multiple (ESCOFIER et PAGES, 1984 et 1988). Les relations entre les différents compartiments de l'écosystème sont plus particulièrement étudiées de façon à juger de l'intérêt de la méthode dans ce cas précis.

\section{MILIEU ET MÉTHODES}

\subsection{Milieu d'étude}

Le ruisseau de Kernec est un affluent de la rive droite du Scorff. Il a déjà été décrit par BAGLINIERE (1979) et HAURY (1985). Ce petit cours d'eau, d'ordre 3 au maximum, a une longueur de 5,6 km pour une largeur moyenne de 1,8 m et une pente de 10,8\%o. L'aval se situe dans une prairie (Prairie de Kernec); puis on trouve une partie forestière (Bois de Kernec). L'amont est plus diversifié du point de vue du boisement des berges.

\subsection{Méthodes d'étude}

Cette étude s'est déroulée en 1981. Les différents compartiments ayant fait l'objet d'analyses antérieures séparées, les techniques sont rappelées brièvement.

\subsubsection{Unités et périodes d'étude}

Le cours principal du ruisseau a été divisé en tronçons consécutifs de $50 \mathrm{~m}$ de longueur sur un total de $3,85 \mathrm{~km}$. Sur chaque unité, ont été réalisés une pêche électrique en octobre, des relevés mésologiques en février et juillet, un inventaire floristique en juillet.

\subsubsection{Etude de la population de truites communes}

Sur chaque secteur, trois classes d'âge ont été distinguées : $0+, 1+,>1+($ comprenant essentiellement des $2+$ et $3+$; voire des $4+$ ). Pour chacune, les effectifs totaux, les tailles moyennes, les effectifs et les pourcentages par sexe ainsi que d'individus mâturants ont été notés.

\subsubsection{Etude mésologique}

En été et en hiver, pour chaque tronçon, ont été mesurés ou estimés :

- les vitesses de courant à $10 \mathrm{~cm}$ du fond (micromoulinet Ott), à l'endroit le plus profond de 5 transects espacés de $10 \mathrm{~m}$,

- les largeurs et profondeurs sur ces mêmes transects, les profondeurs retenues correspondant à la moyenne des maximums,

- les pourcentages approximatifs des substrats apparents, en rajoutant 2 classes organiques racines et débris végétaux - aux classes granulométriques de CAILLEUX (1954),

- le pourcentage de transmission lumineuse au niveau du cours d'eau à midi (mesures au luxmètre par temps ensoleillé). 
Par ailleurs, les pentes du fond du lit ont été mesurées avec mire et niveau sur chaque tronçon. Le pourcentage d'émersion dans le lit mineur a été estimé en juillet.

\subsubsection{Etude de la végétation}

Après inventaire, seules les macrophytes les mieux représentées sur la zone étudiée ont été retenues. Leur recouvrement a été estimé selon une échelle quantitative dérivée de celle des coefficients d'abondance - dominance (GUINOCHET, 1973).

\subsection{Analyse multidimensionnelle}

\subsubsection{Le tableau étudié}

Le tableau analysé est rectangulaire. II comprend 77 individus (secteurs d'étude) et 68 variables (paramètres de description des secteurs). Ces dernières sont réparties en trois blocs:

-22 concernent les truites,

- 26 sont des variables mésologiques,

- 20 correspondent aux recouvrements des principales espèces macrophytiques.

Le seuil du coefficient de corrélation retenu est de 0,23 (ce qui correspondrait à une probabilité de réalisation de $95 \%$, si les variables étaient distribuées normalement).

Tableau I : Valeurs de quelques paramètres par zone écologique.

Nota : Les chiffres entre parenthèses correpondent à l'écart-type des paramètres.

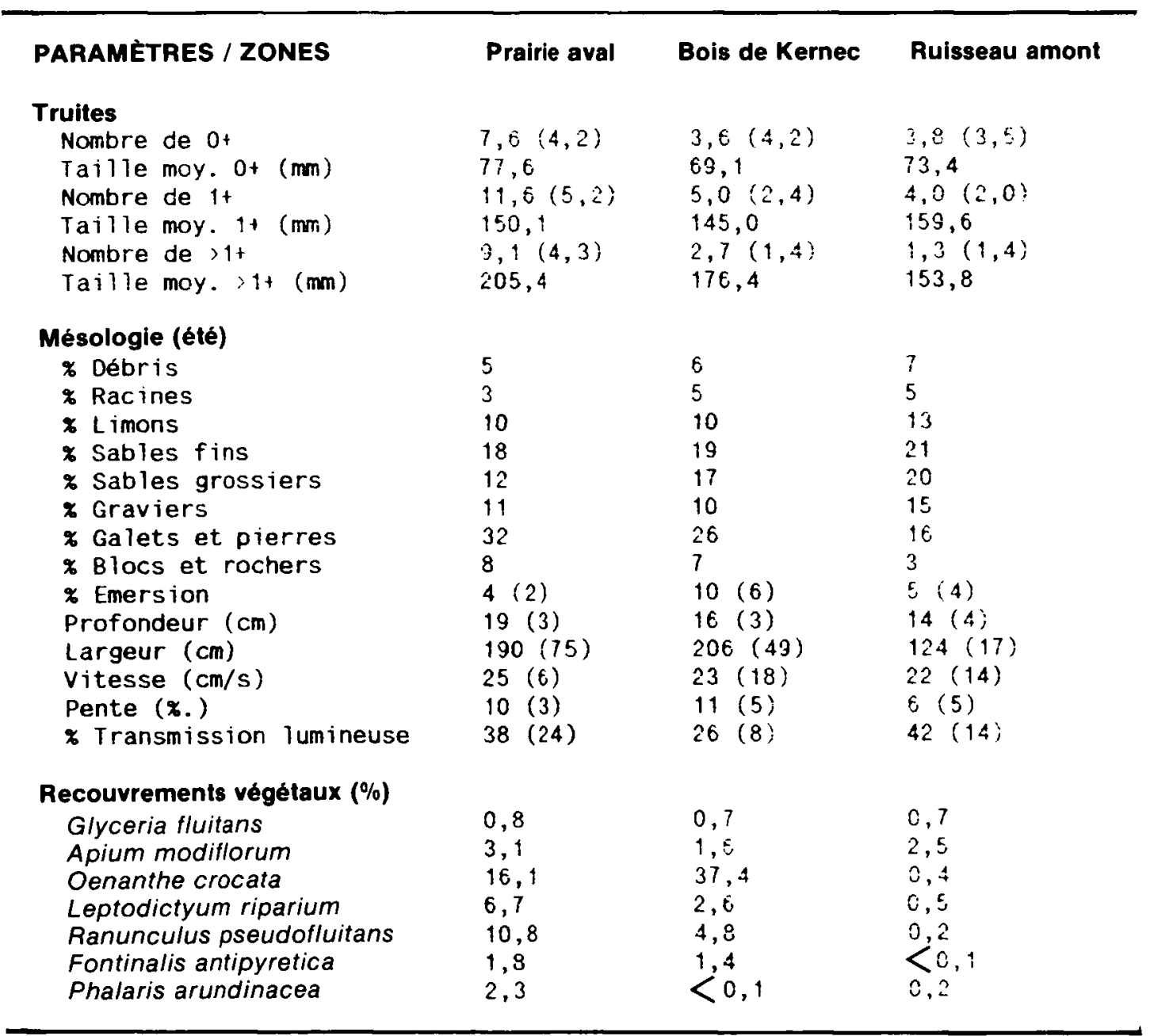


Table I : Values of some parameters for each ecological zone.

Nota: The figures within brackets are the standard deviation for the parameters.

PARAMETERS / ZONES

\section{Trouts}

Number of $0 \mathrm{t}$

Average length of $0+(\mathrm{mm})$

Number of $1+$

Average length of $1+(\mathrm{mm})$

Number of $>1+$

Average length of $>1+(\mathrm{mm})$

\section{Downstream Meadow}

$7,6(4,2)$
77,6
$11,0(5,2)$
150,1
$9,1(4,3)$
205,4

$7,6(4, \hat{2})$

$11,0(5,2)$

$9,1(4,3)$

205,4

$\begin{array}{r}\text { Kernec } \\ \text { Wood }\end{array}$
$3,6: 4,2)$
$63,:$
$5,0(2,4)$
145,0
$2,7(1,4)$
176,4

Kernec

5

5

10

18

12

11

32

8

4 (2)

19 (3)

$190(75)$

25 (6)

10 (3)

$38(24)$

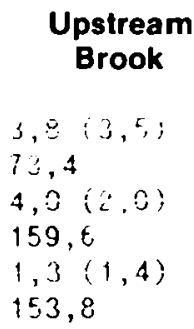

Upstream

Brook

$3,8 \div 3,5 ;$

73,4

$4,3(2,0)$

159,6

$1,3(1,4)$

153,8

\section{Plant cover (\%)}

Glyceria fluitans

Apium modiflorum

Oenanthe crocata

Leptodictyum riparium

Ranunculus pseudofluitans

Fontinalis antipyretica

Phalaris arundinacea

$\begin{array}{lll}0,8 & 0,7 & 0,7 \\ 3,1 & 1,6 & 2,5 \\ 16,1 & 37,4 & 0,4 \\ 6,7 & 2,6 & 0,5 \\ 10,8 & 4,8 & 0,2 \\ 1,8 & 1,4 & <0,1 \\ 2,3 & <0,1 & 0,2\end{array}$

\subsubsection{Choix d'une méthode de traitement des données}

Dans les méthodes multidimensionnelles classiques, Analyse Factorielle des Correspondances et Analyse en Composantes Principales (BENZECRI, 1973), un seul nuage est analysé. Lorsque l'on veut étudier plusieurs compartiments d'un même écosystème, on distingue 2 types de variables: actives et supplémentaires.

Désormais, des méthodes multitableaux permettent d'analyser d'abord séparément puis conjointement des blocs de variables (ESCOFIER et PAGES, 1984; LEBRETON et al., 1988). En Ecologie, les relations statistiques entre les différents compartiments de l'écosystème mais aussi la structure de ce dernier sont donc considérées. L'Analyse Factorielle Multiple (ESCOFIER et PAGES, 1984 et 1985) est l'une d'entre elles; elle a déjà été utilisée en Hydrobiologie par PREVOST (1987) pour étudier les relations captures de saumons-rivières.

Cette méthode consiste d'abord en une analyse multidimensionnelle simple de chaque sous-tableau; comme toutes les variables considerees présentement sont quantitatives, il s'agit d'Analyses en Composantes Principales. Puis une analyse de l'ensemble du tableau est effectuée et les corrélations entre variables calculées. La méthode permet une pondération entre les blocs de variables. La liaison entre les groupes est mesurée ; la projection de ces groupes selon les directions d'inertie maximale les plus communes à l'ensemble des groupes permet d'obtenir une représentation simultanée approchée de chaque groupe de variables. La qualité de la représentation de chaque groupe dans chaque direction (axe factoriel) est définie. 


\section{RÉSULTATS ET INTERPRÉTATION}

\subsection{Les indices généraux de l'Analyse globale}

Le bloc "truites" a une structure bi-dimensionnelle, comme celui des macrophytes ; celui de la mésologie est tri-dimensionnel. Au total, seuls les deux premiers axes ressortent dans l'analyse globale, avec des pourcentages d'inertie de 15,1 et 9,6\%; puis la décroissance est régulière.

Les corrélations entre les variables canoniques (dues à chaque groupe) et les variables générales mettent en évidence une bonne représentation des 3 blocs sur l'axe 1 : la part d'inertie des premiers axes des analyses séparées conservée sur ce premier axe de l'A.F.M. est respectivement de $80,8 \%$ pour le bloc des poissons, $90,8 \%$ pour celui de la mésologie, et $92,2 \%$ pour celui des macrophytes. Sur le second axe, ces pourcentages deviennent : $56,3 \%, 72,4 \%$ et $92,8 \%$. Ceci signifie donc qu'un seul facteur est nettement commun aux trois compartiments de l'écosystème, l'axe 2 étant surtout déterminé par la mésologie et les macrophytes.

\subsection{Analyse des individus et des variables}

\subsubsection{Représentation des variables et signification des axes factoriels}

Pour les variables, l'axe 1 oppose les sables grossiers (aux deux périodes) et les racines (en hiver) d'une part, aux substrats grossiers (pierres, blocs en été), à la pente et aux fortes largeurs, d'autre part (Figure 1)

Les truites participent à son inertie, essentiellement par les individus âgés. On observe une répartition de plus en plus négative des effectifs lorsque l'âge augmente. Pour chaque classe d'âge, les mâles ont des coordonnées plus négatives que les femelles.

Les macrophytes les plus corrélées à l'axe sont situées sur la partie négative : Fontinalis antipyretica, Riccardia sinuata, Leptodictyum riparium, Phalaris arundinacea et Oenanthe crocata. Seules Stellaria alsine et Juncus effusus ont de faibles coordonnées positives.

Du point de vue des variables mésologiques, le premier facteur écologique correspond donc à la stabilité des substrats et à la taille du milieu, ce que corroborent les résultats précédemment acquis sur la répartition des poissons et l'écologie des macrophytes.

Sur l'axe 2, il y a opposition entre, d'une part, le pourcentage d'émersion en été, la largeur hivernale, les débris, pierres et blocs en hiver, et, d'autre part, l'éclairement.

Les macrophytes opposent Phalaris arundinacea, et dans une moindre mesure Sparganium erectum, à Fissidens pusillus, Platyhypnidium rusciforme, Riccardia sinuata et Chrysosplenium oppositifolium auxquelles s'adjoint Oenanthe crocata.

Les truites contribuent à l'inertie de l'axe, même si le compartiment est mal représenté dans l'analyse globale. Ce sont les mêmes variables que celles qui participent à l'axe 1, sans qu'apparaisse la gradation d'ancienneté.

Cet axe est d'interprétation difficile : il est nettement inféodé à la lumière, mais aussi à la largeur et à l'émersion, et aux substrats grossiers. 


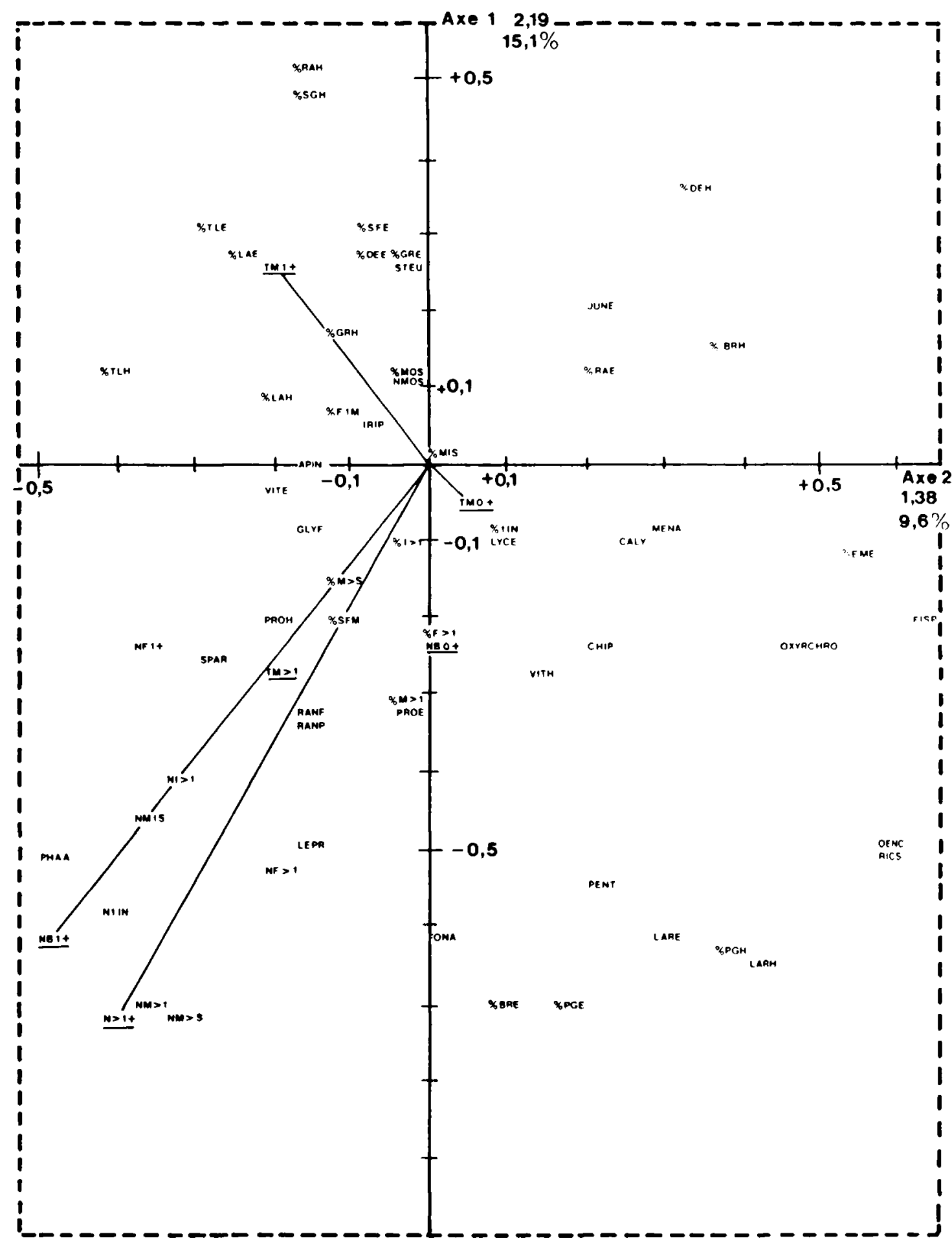

figure 1 : Représentation des variables dans le plan factoriel 1×2

\section{Variables du groupe 1 (truites)}

NBO+ $=$ Nombre de $0+$

TMO+ $=$ Taille moyenne des $0+$

NMOS = Nombre de mâles $0+$ spermiants

$\%$ MOS $=\%$ de mâles $0+$ spermiants
$\% 1$ IN $=\%$ de $1+$ indéterminés

$\mathrm{N}>1+=$ Nombre des plus àgés que $1+(>1+)$

$\mathrm{TM}>1=$ Taille moyenne des $>1+$

NF $>1=$ Nombre des femelles $>1+$ 


$\begin{array}{llll}\text { NB } 1+ & =\text { Nombre de } 1+ & \% \mathrm{~F}>1 & =\% \text { de femelles }>1+ \\ \text { TM } 1+ & =\text { Taille moyenne des } 1+ & N M>1 & =\text { Nombre de mâles }>1+ \\ \text { NF } 1+ & =\text { Nombre de femelles } 1+ & \% M>1 & =\% \text { de mâles }>1+ \\ \% F 1 M & =\% \text { de femelles } 1+\text { mâtures } & N M>S & =\text { Nombre de mâles }>1+\text { spermiants } \\ \text { NM1S } & =\text { Nombre de mâles } 1+\text { spermiants } & \% M>S & =\% \text { de mâles }>1+\text { spermiants } \\ \% M 1 S & =\% \text { de mâles } 1+\text { spermiants } & N I>1 & =\text { Nombre d'indéterminés }>1+ \\ \text { N1IN } & =\text { Nombre de } 1+\text { indéterminés } & \% l>1 & =\% \text { d'indeterminés }>1+\end{array}$

Nota : les pourcentages correspondent à la classe d'âge et non à l'effectif total.

Variables du groupe 2 (mésologie)

\begin{tabular}{|c|c|c|c|}
\hline$\% \mathrm{DEH} / \mathrm{E}$ & $\%$ débris organiques hiver $(H) /$ èté $(E)$ & $\% \mathrm{BRH} / \mathrm{E}$ & $=\%$ blocs et rochers hiver $(H)$ /été $(E)$ \\
\hline$\%$ RAH/E & $\%$ racines hiver $(H)$ /èté $(E)$ & $\%$ EME & $=\%$ èmersion en été \\
\hline$\% \mathrm{LAH} / \mathrm{E}$ & \% limons et argiles hiver $(H)$ /étè $(E)$ & $\mathrm{LARH} / \mathrm{E}$ & $=-$ Largeur hiver $(H) /$ été $(E)$ \\
\hline$\%$ SFH/E & - \% sables fins hiver $(H)$ /éte $(E)$ & $\mathrm{PROH} / \mathrm{E}$ & - Profondeur hiver $(H) /$ èté $(E)$ \\
\hline$\%$ SGH/E & - \% sables grossiers hiver $(H) /$ èté $(E)$ & VITH/E & - Vitesse du courant hiver $(H)$ /été $(E)$ \\
\hline$\%$ GRH/E & - \% graviers hiver $(H)$ /èté $(E)$ & PENT & $=$ Pente \\
\hline$\% \mathrm{PGH} / \mathrm{E}$ & $=\%$ pierres et galets hiver $(H) /$ èté $(E)$ & $\%$ TLH/E & $=\%$ de transmission lumineuse $\mathrm{H} / \mathrm{E}$ \\
\hline
\end{tabular}

Variables du Groupe 3 (macrophytes)

\begin{tabular}{|c|c|c|c|}
\hline APIN & $=$ Apium nodiflorum & LYCE & $=$ Lycopus europaeus \\
\hline CALY & - Callitriche platycarpa & MENA & - Mentha aquatica \\
\hline $\mathrm{CHIP}$ & - Chiloscyphus polyanthos & OENC & = Oenanthe crocata \\
\hline CHRO & -- Chrysosplenium oppositifolium & OXYR & $\therefore$ Platyhynidium rusciforme \\
\hline FISP & - Fissidens pusillus & PHAA & - Baldingera arundinacea \\
\hline FONA & - Fontinalis antipyretica & RANF & = Ranunculus flammula \\
\hline GLYF & = Glyceria fluitans & RANP & $=$ Ranunculus pseudofluitans \\
\hline IRIP & - Iris pseudacorus & RICS & $=$ Riccardia sinuata \\
\hline JUNE & - Juncus effusus & SPAR & $=$ Sparganium erectum \\
\hline LEPR & - Leptodictyum riparium & STEU & = Stellaria alsine \\
\hline
\end{tabular}

Nota : Nomenclatures de Flora Europaea et de "Flore des Bryophytes" d'AUGIER.

figure 1 : Localization of the variables within the plan defined with the factorial axis $1 \times 2$.

Variables of the 1 st group (brown trout)

$\mathrm{NBO}+=0+$ number

TMO $+=0+$ average size

NMOS - Number of $0+$ spermating males

$\%$ MOS $\quad-\%$ of $0+$ spermating males

NB $1+-1+$ number

TM $1+=1+$ average size

NF $1+=1+$ females number

$\%$ F $1 \mathrm{M}=\%$ of $1+$ mature females

NM1S = Number of $1+$ spermating males

$\%$ M1S - \% of $1+$ spermating males

NIIN - $1+$ undeterminated-sex trouts number

Nota: The percentages are expressed within the age-class, and not with the total number of trouts.

Variables of the 2nd group (mesological parameters)

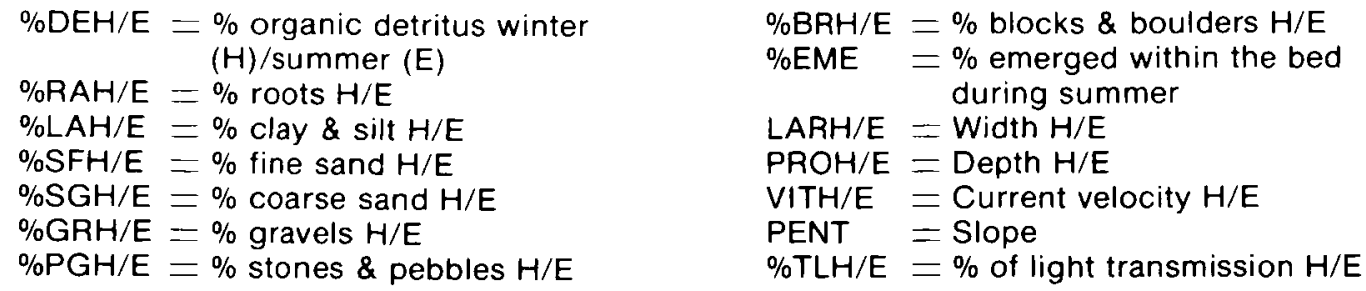


Variables of the 3rd group (macrophytes)

$\begin{array}{ll}\text { APIN } & =\text { Apium nodiflorum } \\ \text { CALY } & =\text { Calitriche platycarpa } \\ \text { CHIP } & =\text { Chiloscyphus polyanthos } \\ \text { CHRO } & =\text { Chrysosplenium oppositifolium } \\ \text { FISP } & =\text { Fissidens pusillus } \\ \text { FONA } & =\text { Fontinalis antipyretica } \\ \text { GLYF } & =\text { Glyceria fluitans } \\ \text { IRIP } & =\text { Iris pseudacorus } \\ \text { JUNE } & =\text { Juncus effusus } \\ \text { LEPR } & =\text { Leptodictyum riparium }\end{array}$

$\begin{array}{ll}\text { LYCE } & =\text { Lycopus europaeus } \\ \text { MENA } & =\text { Mentha aquatica } \\ \text { OENC }=\text { Oenanthe crocata } \\ \text { OXYR }=\text { Platyhynidium rusciforme } \\ \text { PHAA }=\text { Baldingera arundinacea } \\ \text { RANF }=\text { Ranunculus flammula } \\ \text { RANP }=\text { Ranunculus pseudofluitans } \\ \text { RICS }=\text { Riccardia sinuata } \\ \text { SPAR }=\text { Sparganium erectum } \\ \text { STEU }=\text { Stellaria alsine }\end{array}$

Nota: The names are given according to Flora Europaea and to "Flore des Bryophytes" d'AUGIER.

2.2.2. Analyse de la répartition des individus (secteurs) dans le plan factoriel $1 \times 2$ (Figure 2)

La répartition des individus met en évidence une opposition amont (coordonnées positives) - aval (partie négative) sur l'axe 1. Ce dernier correspond donc à un gradient d" importance relative" du cours d'eau.

II y a opposition sur l'axe 2 entre les secteurs éclairés et relativement étroits de la Prairie de Kernec, et les secteurs plus larges et ombragés du Bois de Kernec (moyennes de quelques parametres par grandes zones: Tableau 1). Cet axe correspond donc à une typologie de la moitié aval du ruisseau en fonction de la lumière et de la largeur.

Sur le plan factoriel obtenu en prenant en compte les trois compartiments, on retrouve donc bien les zones du ruisseau qui avaient été définies antérieurement sur les seuls critères physionomiques.

Toutefois, il existe des discordances entre l'image globale des secteurs et celle donnée par chaque compartiment. Quelques exemples représentatifs des secteurs antérieurs d'étude de la truite sont figurés.

\subsection{Résultats complémentaires de la matrice des corrélations}

\subsubsection{Population de truites}

a) Les effectifs

Le nombre des $0+$ est corrélé positivement avec leur taille moyenne, mais ne présente pas de corrélation nette avec les autres classes d'âge. Du point de vue mésologie, on les trouve plutôt sur les substrats grossiers (pierres et graviers en été) et dans les secteurs larges (largeur en hiver); en revanche, ils sont exclus des substrats fins et organiques en été comme en hiver. Les liaisons avec les végétaux sont peu nettes, hormis pour Apium nodiflorum, Sparganium erectum et Ranunculus flammula.

Le nombre des $1+$ est très corrélé à celui des $>1+$, mais il n'existe aucune corrélation entre effectif et taille. En ce qui concerne la mésologie, cette classe d'âge est bien corrélée aux substrats grossiers en été, et aux limons en hiver (ce qui correspond à des zones d'affouillement); en revanche, elle est exclue des biotopes à sables grossiers et des zones où les débris organiques s'accumulent en hiver; la largeur, les profondeurs et la pente sont des facteurs favorables. L'effectif des $1+$ est corrélé positivement avec Phalaris arundinacea $(+0,70)$. Fontinalis antipyretica, Leptodictyum riparium, Sparganium erectum, Glyceria fluitans, et dans une moindre mesure avec Ranunculus pseudofluitans et $R$. flammula. En conclusion, les $1+$ se trouvent dans des milieux plus profonds, éventuellement plus hétérogènes, car la pente plus forte correspond à plus de "cascades".

L'effectif des individus les plus âgés $(>1+$ ) est corrélè à la taille moyenne de la classe d'âge. II existe une corrélation négative avec les racines, débris organiques, sables grossiers et blocs en hiver, et avec les sables grossiers en été ; en revanche, la corrélation est positive avec les sables fins et les pierres en hiver, avec les pierres et blocs en été. Largeurs, profondeurs et pente sont des facteurs favorables. Les corrélations avec les macrophytes sont pratiquement identiques à celles des $1+$, avec des valeurs accentuées avec Fontinalis antipyretica et Ranunculus pseudofluitans, et moindres avec Sparganium erectum et Glyceria fluitans. Il en ressort un peu le même type de preferendum que pour les $1+$.

b) Les tailles

La taille des $0+$ est corrélée négativement avec les sables fins et les vitesses en hiver. positivement avec les graviers en hiver. Les Iris indiquent des biotopes défavorables. 


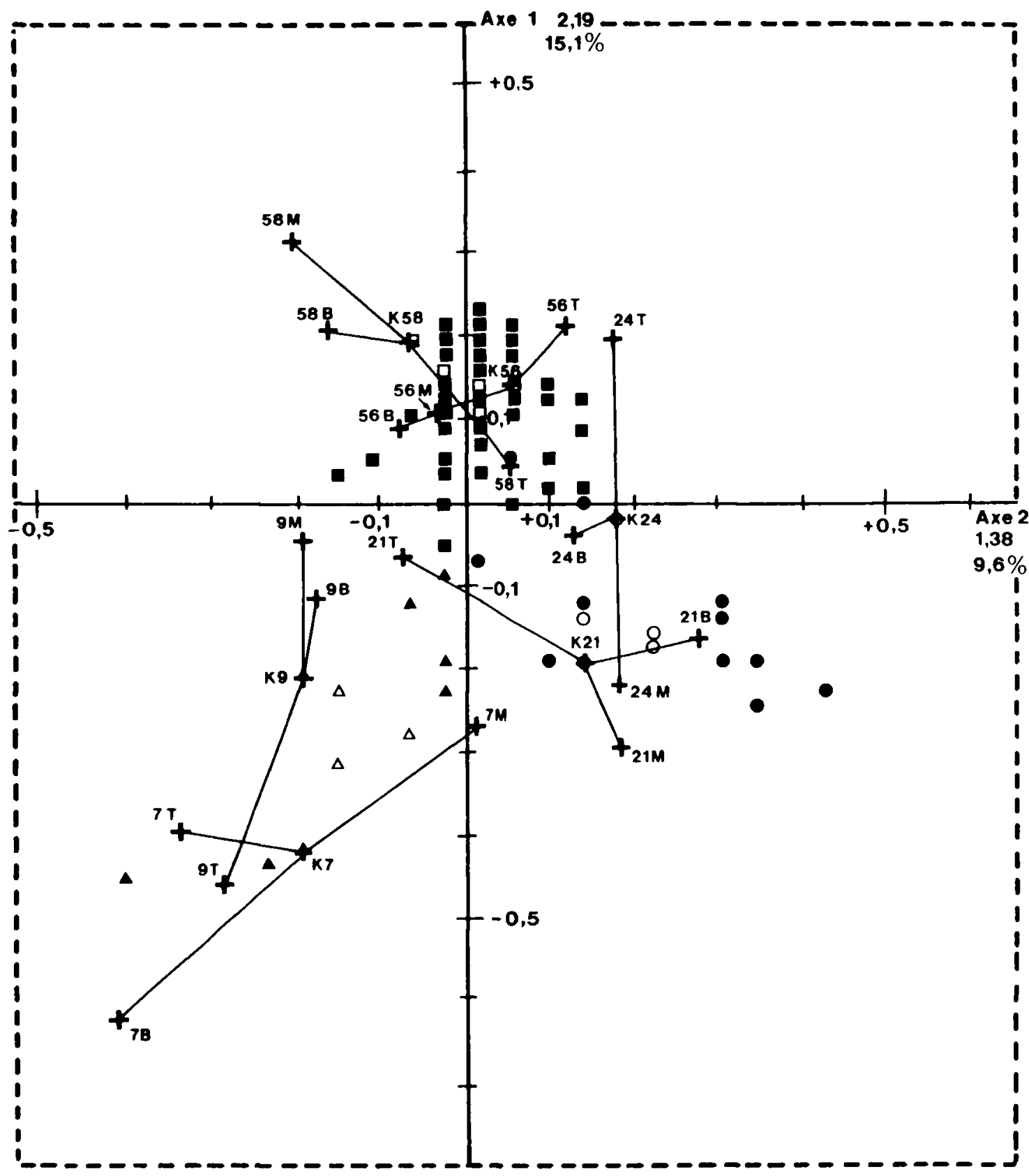

figure 2 : Distribution des secteurs d'étude dans le plan factoriel 1x2.

Les trois grandes zones sont repérées par des figurés différents : triangles pour la Prairie de Kernec; ronds pour le Bois de Kernec; carrés pour l'amont. Les figurés évidés correspondent aux secteurs antérieurs d'étude de la truite. Pour les points extrêmes de ces secteurs d'étude, repérés par les numéros, les images de chaque bloc sont repérées dans le plan factoriel : truites $(T)$, mésologie $(M)$, botanique - macrophytes $(B)$.

figure 2 : Distribution of the studied stretches within the plan defined with the factorial axis $1 \times 2$.

The three main zones are expressed with different symbols : triangles for Kernec Grassland; circles for Kernec Wood; squares for upstream areas. The empty symbols correspond to the permanent stretches for brown trout studies. For the extremum stretches of these permanent stretches, indicated with figures, the projection of each variables group is presented : trouts (T), mesological factors (M), botany - macrophytes (B). 
La taille des $1+$ est faiblement corrélée positivement avec les débris en été, et négativement avec les substrats grossiers (pierres en hiver et blocs en été) et avec la pente. Les milieux à Oenanthe crocata et Chiloscyphus polyanthos - "radiers à Oenanthe" de HAURY (1985) -. sont défavorables.

La taille des individus âgés est faiblement corrèlée avec les sables fins en hiver, les vitesses en été et la pente.

\section{c) L'influence du sexe}

Les femelles $1+$ présentent une corrélation négative avec les racines en été, positive avec les limons en été et en hiver. Les corrélations sont positives avec Apium nodiflorum, Leptodictyum riparium et Phalaris arundinacea.

Les femelles âgées sont défavorisées par les racines, les sables grossiers et les blocs en hiver. par les sables en été; en revanche, les pierres en été comme en hiver, les blocs en été, la largeur importante (mesurée en hiver) et la pente élevée sont des facteurs favorables. Les corrélations sont positives avec Riccardia sinuata, $L$. riparium, $P$. arundinacea, $R$. pseudofluitans et $F$. antipyretica.

Les mâles spermiants $1+$ sont défavorisés par les débris et racines en hiver, les sables grossiers et fins en été; en revanche, on note une corrélation positive avec les pierres et les blocs en été, et avec les espèces végétales suivantes : L. riparium, Ranunculus pseudofluitans, Fontinalis antipyretica; elles sont plus nettement positives avec $P$. arundinacea et nulles avec $A$. nodiflorum.

Par rapport aux femelles $>1+$, les mâles âgés identifiés comme tels sont défavorisés par les débris en hiver, mais favorisés par les sables fins en hiver, la profondeur en hiver plus importante et la largeur en été plus élevée; les corrélations positives avec les pierres en hiver et avec les sables fins en été n'apparaissent plus. Dans la liste des espèces ayant des corrélations positives, Ranunculus flammula et Glyceria fluitans apparaissent et $R$. sinuata est à retrancher. Les mâles spermiants $>1+$ sont situés dans des milieux un peu plus profonds et plus larges que les non-spermiants; les corrélations avec les macrophytes sont comparables.

Les individus $1+$ dont le sexe n'avait pas pu être déterminé présentent des corrélations assez proches de celles des mâles identifiés.

Les "indéterminés" > $>$ t présentent peu de corrélations significatives avec les facteurs mésologiques. On retrouve un rôle défavorable des débris en hiver, positif des sables fins en hiver; la corrélation avec le pourcentage de graviers en hiver est négative. En ce qui concerne les macrophytes, on voit apparaître des corrélations négatives avec Mentha aquatica et Juncus effusus, et positives avec Phalaris arundinacea, Ranunculus pseudofluitans et Fontinalis antipyretica.

\subsubsection{Ecologie des macrophytes}

Seule est analysée l'écologie des espèces présentes dans plus de $20 \%$ des tronçons et ayant des corrélations significatives avec les effectifs ou les tailles des truites.

Glyceria fluitans est nettement inféodée aux limons, mais exclue des sables fins. Les plus fortes largeurs (mesurées en été) lui sont favorables.

Apium nodiflorum est légèrement favorisée par les sables grossiers en hiver et par une forte pente : elle ne pousse pas lorsqu'il y a trop de racines en été (c'est-à-dire sous couvert arboré), mais est abondante sur limons en été.

Oenanthe crocata est également défavorisée par les racines en hiver (ce qui correspondà des zones ombragées et affouillées), par les débris organiques et les limons en été ; en revanche, elle supporte bien les substrats grossiers, pierres en hiver et blocs en été. C'est une espèce favorisée par une pente importante, supportant bien l'émersion, les milieux larges, profonds en hiver mais pas en été ; elle apparaît comme plutôt sciaphile, recherchant l'ombrage en été.

Iris pseudacorus est héliophile, inféodée aux milieux profonds, corrélée positivement avec les sables fins en été, négativement avec les sables grossiers en hiver.

Leptodictyum riparium présente des corrélations négatives avec les substrats instables (débris et sables fins en hiver, sables grossiers en été) et positives avec les substrats stables (pierres et blocs) ; elle est défavorisée par un trop fort éclairement estival. La largeur hivernale élevée est un facteur favorable.

Phalaris arundinacea est corrélée négativement avec les débris en hiver et avec les sables en été, mais positivement avec les pierres et blocs en été ; c'est une espèce corrélée avec la profondeur: elle forme un groupement ripicole partiellement flottant sur l'eau.

Ranunculus pseudofluitans est défavorisée par les racines en hiver, mais présente des corrélations positives avec les pierres, la profondeur et la vitesse du courant en été : c'est une rhéophile qui nécessite une certaine profondeur pour se développer. 
Fontinalis antipyretica est défavorisèe par les limons et sables grossiers en hiver comme en èté, par les graviers en été et par le fort éclairement estival. En revanche, elle est corrélée positivement avec les pierres (et les sables fins) en hiver, avec les pierres et les blocs en été, et avec la pente.

Chiloscyphus polyanthos est inféodée aux pierres en hiver, aux fortes largeurs et à la pente importante; elle est défavorisée par le fort éclairement estival.

\section{DISCUSSION}

\subsection{Les truites et leur habitat}

Les résultats des plans factoriels et de la matrice des corrélations entre les trois classes d'âge, la mésologie et les macrophytes montrent différents phénomènes qui étayent statistiquement certaines hypothèses formulées précédemment, en nuancent quelques autres et corroborent des résultats déjà acquis sur les truites.

- Il existe une zonation longitudinale des classes d'âge, les individus $0+$ étant proportionnellement situés plus en amont (BAGLINIERE et al., 1989), et les poissons plus âgés plus en aval, spécialement les $>1+$ (MAISSE et al., 1987). Cette zonation longitudinale correspond tout à fait à celle des macrophytes (HAURY, 1985), ce qui se traduit par les corrélations "significatives" entre les truites âgées et les végétaux de l'aval.

- L'existence de preferendums physiques semblables à ceux définis dans la rivière principale (BAGLINIERE et CHAMPIGNEULLE, 1982; BAGLINIERE et ARRIBE-MOUTOUNET, 1985) est vérifiée sur le ruisseau : les $0+$ sont situés dans les radiers et les individus âgés dans les milieux plus profonds, avec des caches. L'analyse permet de mettre en évidence des habitats favorables à la fois à la densité et à la croissance pour les $0+$ et les $>1+$. Pour la population de $0+$, une corrélation négative densité-taille avait pourtant été observée par BAGLINIERE et al. (1989), mais dans des conditions d'étude différentes.

- Cependant, pour les $0+$, on ne trouve pas d'habitat optimal à la fois pour la densité et la croissance. Le milieu radier, défini comme le meilleur habitat pour la densité des $0+$, n'est pas optimal pour la croissance, ce qui a été analysé gràce à la matrice des corrélations : en effet, les radiers sont abondants en milieu ombragé, dans le Bois de Kernec (Tableau I). Le meilleur milieu pour la croissance semble se rapprocher de celui colonisé par les $1+$ : ceci implique donc une migration des juvéniles pour une croissance satisfaisante en fonction des nouveaux besoins territoriaux (ALLEN, 1969). Cette migration s'effectuera essentiellement vers l'aval où les $1+$ sont plus abondants, ou bien hors du ruisseau pendant le printemps, ce qui confirme les observations de BAGLINIERE et al. (1989).

- L'absence de relations entre densité et taille chez les $1+$ montre qu'on ne peut déterminer d'habitat préférentiel pour cette classe d'âge. Effectivement, les données montrent une colonisation par cette classe d'âge de l'ensemble du ruisseau, à condition que la granulométrie ne soit pas trop grossière.

- Les très fortes corrélations entre les effectifs de $1+$ et de $>1+$, et entre les macrophytes et la densité de ces deux classes d'âge les placent en aval. Compte tenu des conclusions antérieures de BAGLINIERE et al. (1989), ceci veut dire que les $1+$ de cette zone correspondent aux futurs géniteurs du ruisseau.

- De véritables changements saisonniers des preferendums concernent essentiellement les truites les plus âgees : ces modifications correspondraient au déplacement des habitats de stabulation et d'alimentation en été vers ceux de reproduction en hiver, mais aussi aux changements temporels de l'habitat dus au cycle hydrologique (MILNER et al., 1985). Cette description mésologique réalisée à deux époques permet, à partir d'une seule période de pêche électrique, d'envisager les relations dynamiques entre les truites é leur habitat.

- Les femelles apparaissent être plus inféodées à l'amont du ruisseau que les mâles ou bien elles colonisent des habitats différents. Comme les observations de MAISSE et al. (1987) montrent une absence de ségrégation amont-aval des sexes sur le ruisseau, il semblerait plutôt exister une ségrégation des habitats entre les deux sexes.

- Les corrélations des individus $1+$ non sexuellement mâtures avec les macrophytes et les caractéristiques mésologiques étant très proches de celles observées avec la sous-population mâle, l'hypothèse que ces individus sont des mâles (MAISSE et al., 1987) est confirmée.

\subsection{Regards critiques sur les acquis méthodologiques}

Les acquis méthodologiques concernent à la fois le protocole d'étude et l'intérêt de la mise en œuvre de I'A.F.M. 


\subsubsection{Le protocole d'étude}

Le protocole d'étude, en considérant des unités courtes, éventuellement hétérogènes et pendant une seule année, amène à quelques résultats qui semblent en apparente contradiction avec les observations réalisées par BAGLINIERE et al. (1989). Ceci correspond aux changements d'échelle espace-temps par rapport aux travaux de ces auteurs.

L'échantillonnage systématique réalisé sur le cours principal de Kernec, avec des unités de longueur constante, sans présupposition sur l'homogénéité a plusieurs conséquences.

- Le découpage important permet la mise en évidence des différents niveaux des structures de l'écosystème en considérant à la fois les regroupements de secteurs ou de variables et les discontinuités entre les groupes ainsi définis.

- La hiérarchie des facteurs écologiques généralement utilisés pour décrire les habitats piscicoles (vitesse de courant, granulométrie, hauteur d'eau : MILNER et al. 1985) n'apparaît pratiquement pas dans cette étude. Seuls ressortent nettement, en tant que facteurs analytiques, les pourcentages granulométriques. La lumière, généralement délaissée dans les études, présente d'assez nombreuses corrélations significatives avec les autres paramètres abiotiques, avec les macrophytes et avec les truites. Pour les macrophytes, elle fait ressortir les espèces sciaphiles: Chrysosplenium oppositifolium et surtout les bryophytes Riccardia sinuata et Platyhypnidium rusciforme; un fort éclairement semble constituer un facteur favorable pour la croissance des truites, mais c'est également dans le milieu considéré un bon descripteur synthètique de la "Prairie de Kernec", par opposition au "Bois de Kernec".

Ces difficultés pour conclure sont une conséquence de l'échantillonnage systématique qui mélange des habitats piscicoles, a priori hétérogènes. Par ailleurs, la récolte des données de vitesse et de profondeur sur des transects équidistants, avec une faible densite des observations. est trop ponctuelle pour obtenir une moyenne réllement représentative de l'hétérogénéité au sein de chaque unite. Il aurait fallu multiplier les mesures afin d'obtenir soit des cartes bathymetriques et des vitesses de courant, soit une estimation statistique de la moyenne et de la variance de ces paramètres. En revanche, pour les substrats comme pour la transmission lumineuse, les estimations grossières de pourcentages, réalisées sur l'ensemble du tronçon, s'avèrent plus judicieuses et utilisables.

- Les recouvrements des macrophytes ne considèrent que des classes de valeur qui pourraient être affinées. Par ailleurs, les types écomorphologiques et la position des différentes espèces. dans le courant ou sur les berges, seraient éventuellement à prendre en compte. Les recouvrements des differents types biologiques, notamment les algues, seraient des observations synthétiques complémentaires.

- La seule prise en compte des truites, certes objet principal de l'étude, appauvrit le "bloc poissons" en éliminant les espèces benthiques comme la Loche franche ef le Chabot qui participent au fonctionnement de l'écosystème.

- Un compartiment est complètement absent : celui des invertébrés benthiques.

\subsubsection{Mise en cuvre de l'A.F.M.}

Malgré les limites imposées par le protocole, différents résultats de l'A.F.M. répondent aux objectifs d'une étude pluridisciplinaire.

La prise en compte globale du système et de chaque compartiment permet effectivement d'analyser les relations statistiques entre les compartiments et de déterminer un facteur commun auquel une signification écologique d'importance relative du cours d'eau a pu être attribuée. C'est le premier niveau de structure qui est déterminé. La représentation graphique simultanée permet de "visualiser" les relations entre les variables.

Cette méthode multidimensionnelle a permis de mettre en évidence des hétérogénéités de différente nature, au sein d'un milieu appartenant au même niveau biotypologique au sens de VERNEAUX (1973).

- Trois grands types de milieux sont distingués : la Prairie de Kernec, le Bois de Kernec et l'amont. Ceci corrobore le choix effectué antérieurement des secteurs "représentatifs d'ètude" dans les travaux de EUZENAT et FOURNEL (1976), BAGLINIËRE (1979), NIHOUARN (1983).

- Au sein de chacune de ces grandes zones, une hétérogénéité générale apparaît, traduite par la dispersion des secteurs dans le plan factoriel. La Prairie de Kernec et le Bois de Kernec apparaissent comme plus diversifiés que l'amont.

- La comparaison des images dues à chaque groupe avec l'image globale est également instructive. Si l'on considère les secteurs d'étude des auteurs précités, il y a une très grande concordance des images sur la zone amont, un peu moindre sur le secteur aval de la Prairie de Kernec (granulométrie plus fine dans un profond, et grosses truites), et une assez grande discordance 
entre ces images dans les différentes unités de $50 \mathrm{~m}$ constitutives du secteur d'étude du Bois de Kernec.

Cette hétérogénéité au sein des unités de $50 \mathrm{~m}$ est probablement une des causes pour laquelle les coefficients de corrélation restent assez faibles.

Les relations entre la végétation et la mésologie, envisagées au niveau de ces secteurs, avec une mesure succincte des paramètres abiotiques, sont assez étroites. II en ressort que l'on peut envisager une description complémentaire de l'habitat physique des truites avec les macrophytes, à trois niveaux:

- pour limiter la quantité de données physiques à récolter,

- pour placer les limites des habitats.

- pour intégrer les variations temporelles, de granulométrie, de profondeur et de vitesse de courant et d'éclairement.

L'habitat du poisson et ses preferendums de densité ou de croissance apparaissent donc liés d'une manière ou d'une autre à l'écologie des macrophytes, ce qui justifie une meilleure intégration de ce compartiment dans les descriptions d'habitat, non seulement en terme de couverture végétale, mais aussi en considérant les espèces dominantes.

\section{CONCLUSION}

Dans cette étude, différentes relations statistiques sont mises en évidence. Toutefois, si certaines réponses biologiques sont supportées sur l'existence des preferendums, certaines questions restent en suspens : analyse de la mobilité dans l'espace et le temps des truites, détermination du fondement écologique des relations statistiques mises en évidence entre les compartiments, ce qui impliquera des phases d'expérimentation et des modifications des études réalisées in situ. Par ailleurs, une prise en compte des invertébrés benthiques serait nécessaire pour analyser les relations trophiques dans le cours d'eau.

Un changement de méthode, en considérant des unités homogènes de surface variable, pourrait constituer une amélioration substantielle du protocole. Cependant, les problèmes de la définition des unités à prendre en compte et de leurs limites risquent d'introduire de nouveaux biais (tronçons tels qu'un radier, ou zone de courant différenciée de la bande située près d'une berge d'affouillement à sous-berge, ou à proximité d'une ceinture à Phalaris, ...). Les surfaces à considérer seraient peut-être l'"individu d'association phytosociologique" (c'est-à-dire l'ensemble des végétaux présents dans l'aire minimale sur laquelle se pratique le relevé phytosociologique en un site déterminé - GUINOCHET 1973) de phanérogames pour le poisson, mais plutôt la "touffe" d'hydrophytes ou de bryophytes pour les invertébrés benthiques. La concordance ou plutôt l'imbrication des échelles d'étude et des niveaux d'hétérogénéité sont autant de difficultés à surmonter.

En raison des relations existant entre flore et mésologie, la prise en compte des peuplements macrophytiques et de la distribution des principales espèces peut éventuellement réduire l'intensité d'échantillonnage mésologique en aidant à placer les limites des unités homogènes.

En définitive, la mise en œuvre de l'Analyse Factorielle Multiple s'avère intéressante pour des études interdisciplinaires afin d'aboutir à une meilleure compréhension du fonctionnement des cours d'eau.

\section{BIBLIOGRAPHIE}

ALLEN K.R., 1969. Limitations on production in salmonid populations in streams. The University of British Columbia, Institute of Fisheries, HR MacMillan Lectures in Fisheries. Symposium on Salmon and Trout in Streams, 1968, 3-18.

BAGLINIERE J.L., 1979. Les principales populations de poissons sur une rivière à salmonidés de Bretagne-Sud, le Scorff. Cybium $3^{\mathrm{e}}$ série, $7: 53-74$.

BAGLINIERE J.L., ARRIBE-MOUTOUNET D., 1985. Microrépartition des populations de truite commune (Salmo trutta L.), de juvénile de saumon atlantique (Salmo salar L.) et des autres espèces présentes dans la partie haute du Scorff (Bretagne). Hydrobiologia, 120:229-239.

BAGLINIERE J.L., CHAMPIGNEULLE A., 1982. Densité des populations de truite commune (Salmo trutta L.) et de juvéniles de saumon atlantique (Salmo salar L.) sur le cours principal du Scorff (Bretagne) : preférendums physiques et variations annuelles (1976-1980). Acta Oecologica, Oecol. Applic., 3 : 241-256.

BAGLINIERE J.L., MAISSE G., LE BAIL P.Y., NIHOUARN A., 1989. Population dynamics of brown trout (Salmo trutta L.) in a tributary in Brittany (France) : spawning and juveniles. J. Fish Biol. 34, 97-110. 
BAGLINIERE J.L., MAISSE G., LE BAIL P.Y., PREVOST E., 1987. Dynamique de la population de truite commune d'un ruisseau breton (France). II. - Les géniteurs migrants. Acta Oecologica, Oecol. Applic,. $8: 201-215$.

BENZECRI J.P., 1973. L'Analyse des Données. 2 vol., Dunod, Paris, 625 p. + 632 p.

CAILLEUX A., 1954. Limites dimensionnelles des noms des fractions granulométriques. Bull. Soc. Géol. Fr., 4 : 643-646.

CHAMPIGNEULLE A., 1978. Caractéristiques de l'habitat piscicole et de la population de juvéniles sauvages de saumon atlantique (Salmo salar L.) sur le cours principal du Scorff (Morbihan). Thèse $3^{\circ}$ Cycle, Biol. Anim, Fac. Sci., Univ. Rennes, 1 vol., 92 p.

ESCOFIER B., PAGES, J., 1984. L'Analyse Factorielle Multiple. Cahiers du Bureau de Rech. Opérationnelle, Sér. Rech., $42: 68 \mathrm{p}$.

ESCOFIER B., PAGES, J., 1985. Mise en œuvre de l'Analyse Factorielle Multiple pour des tableaux numériques, qualitatifs ou mixtes. Publ. Interne IRISA, 263 : 56 p.

ESCOFIER B., PAGES J., 1988. - Analyses factorielles simples et multiples - Objectifs, méthodes et interprétation. Dunod ed., Paris, 1 vol., 241 p.

EUZENAT G., FOURNEL F., 1976. Recherches sur la truite commune (Salmo trutta L.) dans une rivière de Bretagne, Le Scorff. Thèse $3^{e}$ Cycle, Biol. Anim., Fac. Sci., Univ. Rennes, 1 vol., 213 p.

GUINOCHET M., 1973. Phytosociologie. 1 vol., Masson, Paris, 227 p.

HAURY J., 1982. Quelques méthodes d'étude de la végétation en écosystème dulçaquicole courant - Application au réseau hydrographique du Scorff, Bretagne. Sci. Agron. Rennes, 1982-2 : 17-33.

HAURY J., 1985. Etude écologique des macrophytes du Scorff (Bretagne-Sud). Thèse de DocteurIngénieur, Ecol., Fac. Sci., Univ. Rennes, 1 vol., 243 p.

LEBRETON J.D., CHESSEL D., PRODON R., YOCCOZ N., 1988. L'analyse des relations espècesmilieu par l'analyse canonique des correspondances. I. - Variables de milieu quantitatives. Acta Oecologica, Oecol. Applic., $9: 53-67$.

MAISSE G., BAGLINIERE J.L., LE BAIL P.Y., 1987. Dynamique de la population de truite commune d'un ruisseau breton (France) : les géniteurs sédentaires. Hydrobiologia, 148 : 123-130.

MILNER N. J., HEMSWORTH R.J., JONES B.E., 1985. Habitat evaluation as a fisheries management tool. J. Fish Biol. (Supplementum A) : 85-108.

NIHOUARN A., 1983. Etude de la truite commune (Salmo trutta L.) dans le bassin du Scorff (Morbihan). Démographie, reproduction, migrations. Thèse $3^{\circ}$ cycle, Ecol., Fac. Sci, Univ. Rennes, 1 vol., 73 p.

PREVOST E., 1987. Recherches sur le saumon atlantique (Salmo salar L.) en France. Thèse de Docteur-Ingénieur, Sci. Agron. Halieutique, Ec. Nat. Sup. Agron. Rennes, 1 vol., 103 p.

VERNEAUX J., 1973. Cours d'eau de Franche-Comté (Massif du Jura). Recherches écologiques sur le réseau hydrographique du Doubs. Thèse d'Etat Sciences, Fac. Sci. Tech., Besançon, 1 vol., $257 \mathrm{p}$. 